\title{
Analyzing the longitudinal impact of proximity, location, and personality on smartphone usage
}

\author{
Lei Meng, Shu Liu and Aaron Striegel*
}

\section{*Correspondence: striegel@nd.edu Department of Computer Science and Engineering, University of Notre Dame, Notre Dame, IN 46556, USA}

\begin{abstract}
Background: Over the past few years, mobile devices, particularly smartphones have seen dramatic increases in data consumption. The significant increases in data usage have placed tremendous strain on the wireless infrastructure, necessitating research across a variety of optimization, efficiency, and capacity improvements.

Complementary to those research efforts is the acquisition of a better understanding of what aspects drive user smartphone usage.
\end{abstract}

Methods: In this paper, we leverage the unique characteristics of the NetSense study, a longitudinal study covering three years of a large cohort of smartphone users, to demonstrate how proximity, location, and individual differences (e.g., personality) can play an important role in understanding smartphone usage over time. We analyze the longitudinal impact of proximity density, personality, and location on smartphone traffic consumption.

Results and Discussion: We show that friendship proximity (i.e., SMS contacts and Facebook friendship) has a significant impact on traffic consumption and personality tends to impact application preference / consumption; However, the true behavior might not be presented due to restrictions of network connectivity. Applications can have significantly different contextual usages based on the location.

Conclusions: We believe our study raises the importance of considering proximity, personality, and location as context relevant for the purposes of assessing user data consumption.

Keywords: Proximity; Location; Smartphone; Personality traits

\section{Background}

With the increasing popularity of smartphones and the wide range of applications available on mobile devices, wireless traffic has experienced tremendous growth in the past few years [1]. Users are able to browse the web, access e-mails, participate in online gaming, and enjoy high-definition multimedia all from their mobile devices. The innate mobility of the said devices affords users the freedom to experience pervasive connectivity across a variety of contexts and environments. As a result, mobile devices are carried nearly ubiquitously from the moment one wakes up to when one goes to sleep, all the while consuming ever-increasing quantities of data. The net result is a seemingly unrelenting demand for data that places tremendous strain on the existing wireless infrastructure [2].

(C) 2014 Meng et al.; licensee Springer. This is an Open Access article distributed under the terms of the Creative Commons

Attribution License (http://creativecommons.org/licenses/by/4.0), which permits unrestricted use, distribution, and reproduction in any medium, provided the original work is properly credited. 
While the process of observing cellular data traffic has gained in popularity significantly in the research community [3-5], the extraction of useful design parameters requires an understanding that can only be gained by observing the overall context of usage. Notably, it is the device usage as impacted by non-smartphone aspects including internal factors (goals, personality) and external factors (friendship, environment, nearby users) that are difficult to monitor but yet potentially quite informative $[5,6]$.

To that end, we leverage the unique characteristics of our NetSense dataset, a longitudinal study covering 3 years of a large cohort of smartphone users at the University of Notre Dame, to offer insight on the said aspects. Our dataset and the associated survey/metadata allow us to explore the interplay of user proximity, friendship, location, and personality type on device and application usage.

The key contributions of our paper are as follows:

- We analyze the impact of user density (i.e., the number of nearby mobile devices) on data consumption and application usage. Further, we assess a variety of friendship filtering methods ranging from simple proximity [7] to more sophisticated friendship criteria (prior short message service (SMS) interactions, prior Facebook interactions, etc.). We find that the context of user proximity plays a significant role.

- We study the impact of personality on traffic consumption and application distributions. We find that personality type tends to influence the top application used, but notably, that such differences only manifest themselves with sufficient connectivity speeds. Under restrictions on network connectivity, smartphone usage is likely restricted as well.

- We analyze the impact of location and how location interplays with application usage/consumption. While it is not surprising that application usage tends to vary with location, the notable finding is that the impact due to the proximity of other users can vary significantly based on location.

\section{Related work}

The study of phone and user characteristics has seen considerable growth over the past few years with numerous studies and smartphone test beds [8-12]. Similar to our own work, many studies have explored a variety of contextual implications for usage including location, proximity, and user interest. The MIT Reality Mining project was one of the first studies to explore the interplay of applications and social interactions [7]. Later studies such as those by Do et al. subdivided usage further into hourly assessments and further refined proximity through the improved use of Bluetooth density [3]. Conversely, others such as $\mathrm{Xu}$ et al. aggregated significantly larger datasets to explore application usage at a national level [12]. Shafiq et al. continued a similar trend by looking at geographical dynamics of application usage [13]. Alternatively, Rahmati and Zhong studied the smartphone usage of 14 novice teenage users [14], and they showed that usage is highly mobile and location dependent.

Several other studies focused on exploring the factors that would influence app usage including individuals' preferences and history, user activities and environment, and also, the app usage of the community a user belongs to [15-18]. In contrast, our own dataset draws from the strength of not only fine-grained longitudinal data (order of minutes, nearly complete contextual logging) but also from significant improvements to Bluetooth 
proximity [19], detailed survey/metadata monitoring from social networks (Facebook, contact lists), and survey data (alter disambiguation, etc.). Although Stopczynski et al. collected a variety of similar data points to the NetSense study including face-to-face interactions, telecommunication, social networks, location, and background information from a larger population of nearly 1,000 users [20], their work focused primarily on analytics with respect to social communications and social network characteristics.

Besides external factors, individual differences such as personality are also non-trivial for data analysis. Although less explored than smartphone context/usage, there are several recent works that have begun to explore linkages between smartphone usage and personality $[5,21]$. Chittaranjan et al. continued the earlier work of Do et al. to analyze the relationships between smartphone usage such as application usage (frequency), calls, SMS messages, and Big-Five personality traits. In [5], Chittaranjan et al. were able to infer the personality type of a user based on aggregated creatures obtained from the smartphone usage data significantly above chance and up to $75.9 \%$ accuracy. In the work by Butt and Phillips [21], the researchers found that disagreeable extraverts reported spending more time calling, changing ringtones, and changing phone wallpaper. Unconscientious, emotionally unstable, disagreeable extraverts also reported spending more time sending text messages. Critically, all data reported by the study was gathered via self-reported surveys in contrast to our own which was directly logged on the device. The key contribution of our work relative to the works by Chittaranjan et al. [5] and Butt and Phillips [21] is to bring location context and user proximity into consideration.

\section{Dataset}

Our data comes from the NetSense study launched in August of 2011 consisting of 200 incoming freshmen at the University of Notre Dame. Each participant in the NetSense study was provided with a free smartphone (Nexus S) with unlimited data, unlimited texting, and unlimited mobile-to-mobile minutes in exchange for complete monitoring access of the phone. As part of the study, a user-level agent was deployed to collect a wide variety of information including network traffic, application traffic, location, phone call, text message, e-mail, browser history, screen usage time, and various other aspects. We note that actual data contents were not logged but rather that only the handset environmental view and who/where/when of phone communications were recorded (e.g., metadata). From an operational perspective, the agent locally saves data onto the local phone storage and then periodically relays the data across a secure connection to the collection server. The full details with regard to the collection mechanisms can be found in [19].

In the August of 2013, the participants were offered the opportunity to upgrade their phones to a set of newer models such as the Galaxy S4, the Galaxy S3, and the HTC One. The newer devices are Android devices as well but add the support of long-term evolution (LTE) and improved WiFi performance. As with many longitudinal studies, various participants had left the study leaving 120 participants after adding a new, small cohort of 20 users. The data relevant to this paper includes the following:

- Bluetooth proximity: A key characteristic of the NetSense study is the active recording of proximity gleaned through Bluetooth discovery. Each participant must leave their device as permanently Bluetooth discoverable, allowing for low-power discerning of relative proximity. Proximity is recorded once every $3 \mathrm{~min}$. 
- WiFi Access Points: WiFi access points (APs) are actively recorded every 3 min via WiFi discovery including station ID, MAC address, and RSSI.

- Phone usage: Phone usage is defined as the time when the phone screen is active, i.e., the user is highly likely to be actively using the phone. Phone usage is recorded by a trigger capturing the exact time when the screen activates until when the screen shuts off.

- Network usage: Students in this study may consume mobile data either through campus-wide WiFi (802.11n) or 3G EVDO (Sprint)/WiMAX (4G). All the newer models upgraded after August 2013 had full LTE support. We log four types of traffic once per minute: total downlink traffic, total uplink traffic, cellular downlink traffic, and cellular uplink traffic.

- App usage: The network usage of each application installed on the phone is logged including the uplink and downlink traffic once per minute. App usage is not broken out by adapter, only total consumption (uplink, downlink).

- Location data: Location is recorded via the Google Android Location Service triggered either by movement out of a perceived 100-m area or if 10 min has elapsed since the last recording. Due to power constraints, GPS is not used but rather WiFi fingerprints and cellular triangulation ascertain an approximate position.

In this paper, users were filtered to include only the instances with active users. An active user is defined as any user with at least $10 \mathrm{~min}$ of screen on time per day. Users are evaluated each day across the entirety of the study for inclusion. Table 1 shows the number of users and days we analyzed at different semesters from August 2011 until spring 2014. Fall semesters are time periods from the start of August to the end of November, and spring semesters span from the beginning of February to the end of May. Summer and winter breaks are not considered since the majority of the students are not on campus. The last two semesters (5-6) see decreased participants by virtue of significant study abroad enrollment that typically occurs in the Notre Dame junior year. Table 1 also summarizes other daily usage of the smartphone per person at different semesters. While the average screen time across different semesters is similar, the traffic usage of semesters 5-6 experiences a significant increase due to the better network connectivity resulting from upgrading to newer models [22]. For the purpose of this paper, we only focus on the downlink traffic in the analysis.

\section{Methods}

In this paper, we aim to explore on the longitudinal impact of three potential factors on smartphone usage including 1) proximity density, 2) personality, and 3) location categories.

Table 1 Data summary

\begin{tabular}{lllllll}
\hline & $\mathbf{1}$ & $\mathbf{2}$ & $\mathbf{3}$ & $\mathbf{4}$ & $\mathbf{5}$ & $\mathbf{6}$ \\
\hline Time period & Fall 2011 & Spring 2012 & Fall 2012 & Spring 2013 & Fall 2013 & Spring 2014 \\
Number of users & 161 & 147 & 125 & 106 & 59 & 60 \\
Number of days & 5,784 & 7,303 & 4,261 & 3,298 & 1,338 & 1,665 \\
Average downlink traffic (MB) & 25.16 & 27.86 & 21.60 & 31.82 & 133.30 & 103.57 \\
Average screen time (hour) & 3.07 & 2.39 & 2.50 & 2.71 & 3.22 & 2.67 \\
\hline
\end{tabular}

The average values are averaged over person and day. 
Bluetooth is a wireless technology in modern wearable devices such as smartphones and tablets for short range communication. The intuition is that two smartphone users in Bluetooth range have the potential for face-to-face interactions (e.g., talking) or to be involved in common activities. Although this proxy is not perfect, Bluetooth proximity has been successfully applied in sensing social interactions and capturing human mobility $[23,24]$. We then use Bluetooth to explore the impact of proximity on smartphone behavior in terms of traffic consumption. Critically, we explore how the impact may vary when a close friend is nearby versus when a stranger sits over at the next table. In short, we further analyze the influence from different types of social relationships.

The Big-Five personality traits are five broad categories that are used to describe human personality including openness, extraversion, neuroticism, agreeableness, and conscientiousness. We use personality traits to examine how individual differences relate to smartphone behavior in terms of traffic usage. Each trait represents a range between two extremes of one to five with most individuals tending away from the extremes. We measure the Big-Five personality traits of each participant by averaging the results from multiple questionnaires the participants take during the study. For a given personality trait, five groups are formed with each of the five scores. For the cases that contain few people (less than 10), we combine them with others having the closest score. For example, the majority of the participants have scores of 3 or 4 for agreeableness, thus only two groups (i.e., $\leq 3$ and $\geq 4$ ) are formed. For each group, we measure the average active traffic at different semesters.

Finally, traffic consumption may occur any time and any places due to the pervasive nature of wireless access for the smartphone. Different location categories may have different usages. For example, the dormitory (dorm) is a place to sleep/interact socially, the classroom is a place to study/have lectures, and the dining halls are places to eat. The different functionality of each location impacts the smartphone usage and thus the traffic consumption. Therefore, it is important to understand the impact of location. Here, we aim to study whether smartphone users have preferences with regards to applications at different locations. To that end, we identify and aggregate the two top location categories - dorm and classroom, where our participants consumed the most of the traffic. We analyze several representative/popular applications including Facebook, the Android Browser, ESPN, and Pandora at different semesters.

\section{Results and discussion}

\section{Proximity density}

A Bluetooth device detected via Bluetooth discovery imparts a signal strength which can roughly indicate the distance between the two devices [19]. It has been shown that devices detected with signal strength less than $-80 \mathrm{dBm}$ are largely due to randomness [19]; thus, we use $-80 \mathrm{dBm}$ as cutoff. We use none (i.e., zero), single (i.e., one), and multiple (i.e., more than one) to refer different proximity densities which represent the number of distinct devices detected in one time slot (5 min).

Figure 1 shows the probability distribution of the occurrence of none, single, and multiple devices in Bluetooth proximity across a day. For each type of proximity density, the probability across all hours adds up to 1 . For example, $7.5 \%$ of the occurrence when multiple device $(\geq 2)$ in proximity happens around 11 AM. The occurrence of multiple devices in proximity is higher in the day and lower during the night since students go to classes 

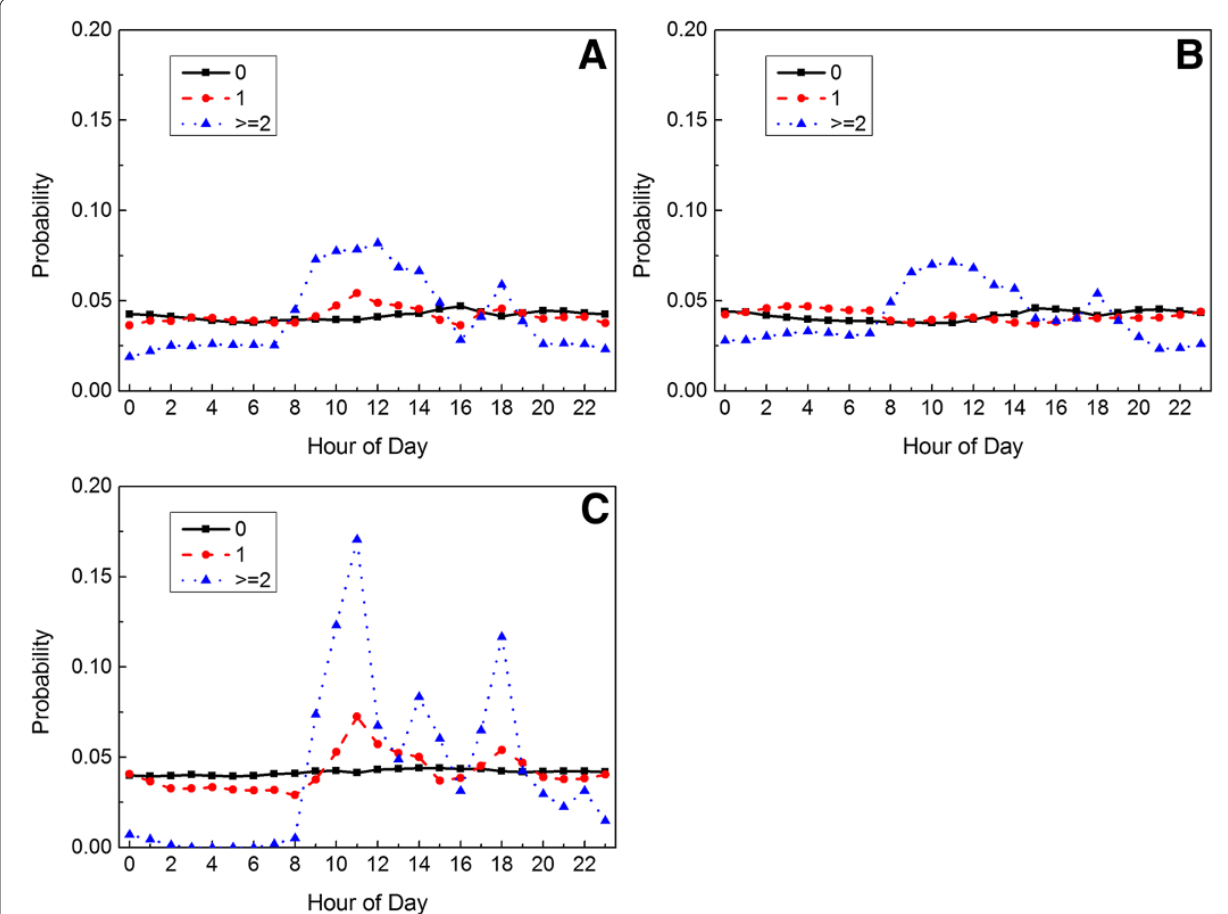

Figure 1 Diurnal patterns for different Bluetooth proximity densities. The diurnal patterns for the none (i.e., 0), single (i.e., 1), and multiple cases (i.e., $\geq 2$ ) at (A) semesters 1-2, (B) semesters 3-4, and (C) semesters 5-6. Each point represents the contribution of a given time slot with respect to that proximity category.

and attend activities mostly in the daytime and go back to the dormitory to rest or sleep in the night. Notably, it peaks around noon and 6 PM which are lunch and dinner time, respectively. The occurrences of zero proximity and single device proximity are similar across the day. For the analysis in this paper, we do not consider the time falling between $12 \mathrm{AM}$ and $8 \mathrm{AM}$ to avoid introducing bias when significant portions of the participants may be sleeping.

For the purpose of traffic analysis, we measure the average active traffic by dividing the total traffic consumed when the screen is on within a period by the number of time slots in that period. The filtering for screen on traffic helps to characterize active use despite nearby users rather than simple background traffic (e.g., Facebook newsfeed). We further refine proximity to 'meaningful' proximity from a social relationship perspective to explore whether friends in proximity play a different role in shaping your smartphone behavior. Two meaningful proximity definitions are SMS contacts being in proximity and Facebook friends being in proximity [25-27]. A SMS contact refers to two people who send or receive at least one text message to each other per month on average during the entire semester. A Facebook friend indicates that the users are on each other's Facebook friend list.

We compare the average active traffic consumed when SMS contacts and Facebook friends are nearby with two other broad categories of proximity (Any - any detected Bluetooth device and Project - only NetSense devices via Bluetooth) as shown in Figure 2. Each bar represents the average active traffic and $95 \%$ confidence interval at a given proximity density based on certain meaning for the first four semesters. For example, in semester 1 , the third bar from the left at single proximity density represents the average active traffic 

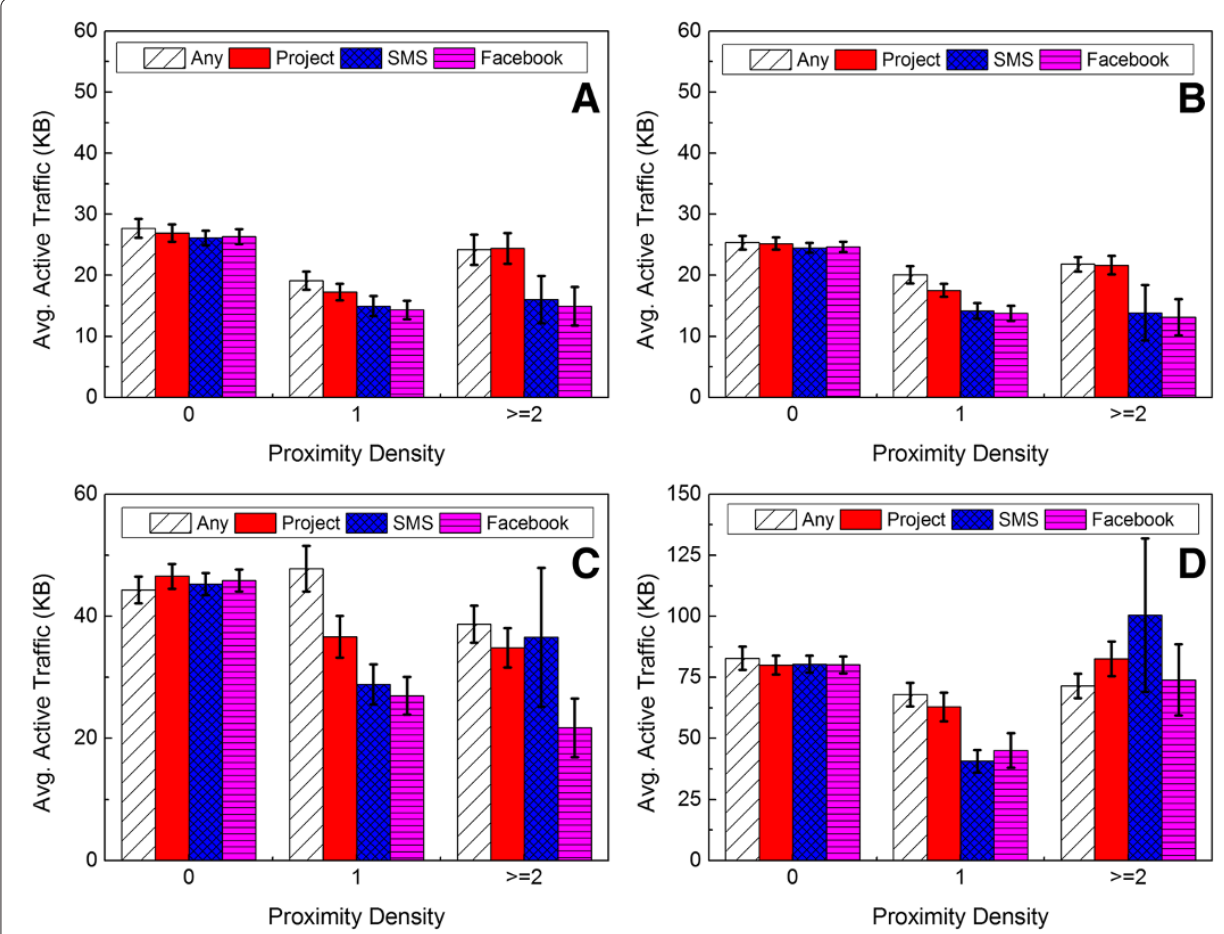

Figure 2 Average active traffic for different Bluetooth proximity densities. Four types of proximity are compared including any (i.e., any detected Bluetooth device), project (i.e., only NetSense devices via Bluetooth), SMS friends (i.e., send or receive at least one text message per month on average), Facebook friends (i.e., on Facebook friend list) at (A) semester 1, (B) semester 2, (C) semester 3, and (D) semester 4.

of a device consumed when one SMS friend is in proximity. The zero (or none) proximity density based on SMS contacts refers to the case when no SMS contacts are in proximity. The average active traffic is not significantly impacted by the number of any/project devices in proximity. However, when single or multiple SMS contacts/Facebook friends appear in proximity, users tend to have much less active traffic on average. This indicates that meaningful proximity relates to the smartphone users' behavior while not discerning such meaning would miss the entire picture.

In semesters 5-6, the quality of the WiFi has improved tremendously while students were less compliant in turning on their Bluetooth. If two devices share multiple access points observed via WiFi scan within a small time slot, the devices are considered in WiFi proximity. Previous work has suggested that devices lacking common WiFi APs are highly unlikely to be in physical proximity $[20,28]$. In order to take advantage of the availability of WiFi proximity, we combine the Bluetooth proximity with WiFi proximity and study whether the combined proximity offers comparable insights in the analysis of smartphone usage. Specially, two devices in combined proximity indicate that one device is in Bluetooth proximity, WiFi proximity, or both of the other device. Here, we further restrict two devices to be in WiFi proximity if they share at least two or more APs.

For any two devices sharing any APs, we look at the distribution of the number of APs the devices shared as shown in Figure 3 at different semesters. For the first two semesters, over $75 \%$ of pairs shared only a single AP while pairs sharing only a single AP for semesters 3-4 decreased by half. The shift in part can be attributed to changes in dormitory/roommates and changing of typical classrooms to smaller venues. For the last 


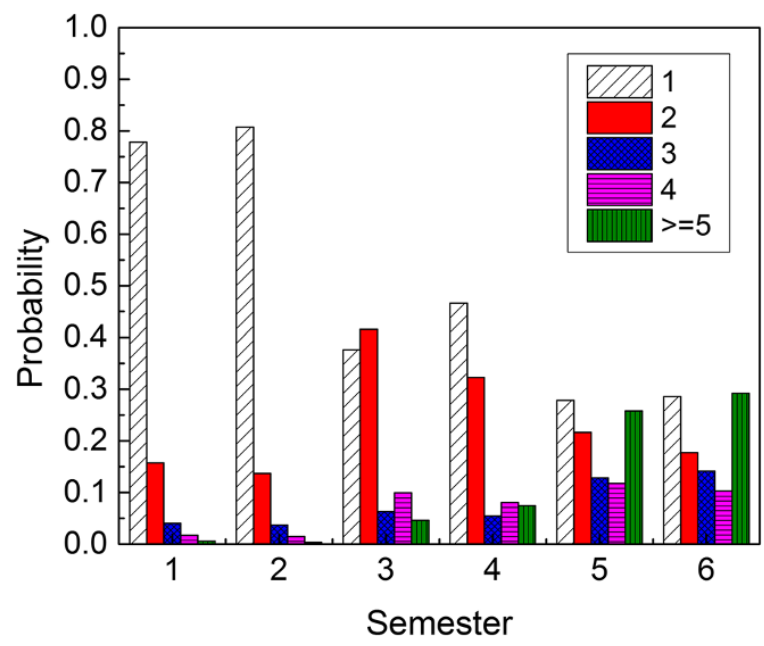

Figure 3 The probability of different numbers of shared APs between any two devices sharing common APs.

two semesters, as the network connectivity of the new handsets got significantly better, around 70\% pairs shared at least two APs when common APs were observed.

In order to understand the composite of combined proximity, we break the combined proximity down by its sources - Bluetooth proximity, WiFi proximity, Bluetooth and WiFi proximity, and Bluetooth or WiFi proximity as shown in Figure 4A. The set of Bluetooth or WiFi proximity is the super set of the set of the other three sources. Each bar represents the probability of proximity coming from a given source out of Bluetooth or WiFi proximity. For the first four semesters, Bluetooth proximity contributed over $80 \%$ while WiFi proximity contributed over $50 \%$ for the last two semesters. WiFi proximity played a more important role among combined proximity for the last two semesters. Next, we look at how often proximity occurs. Figure 4B shows during the day the probability of occurrence of at least one project devices in proximity broken down by the source of the proximity. At all semesters, at least $10 \%$ of the time during a day a project device would in proximity from Bluetooth or WiFi. The decreased probability of proximity at later semesters was
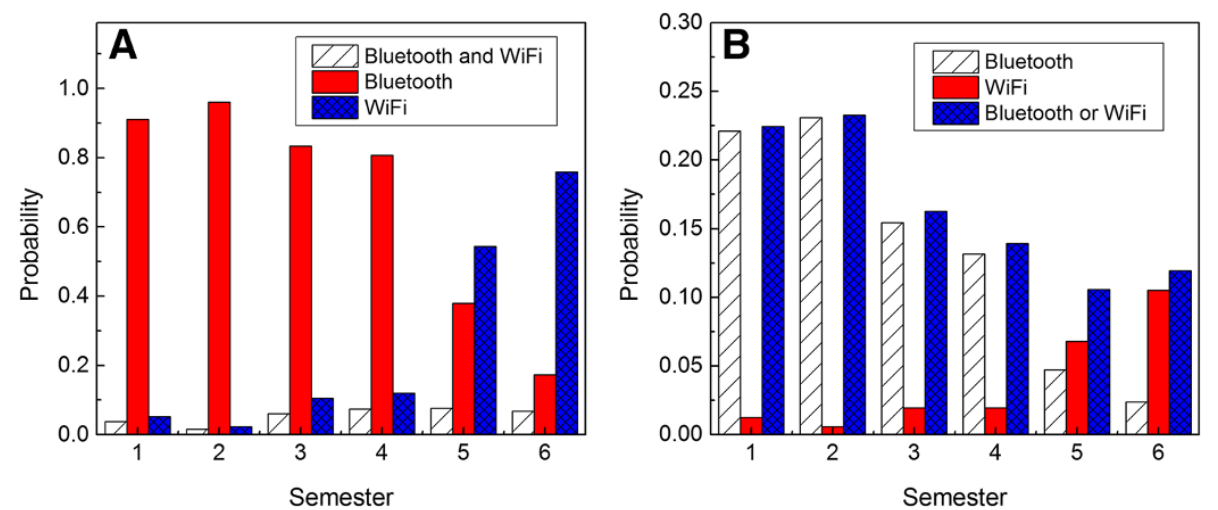

Figure 4 Probability of different sources of proximity. (A) The probability of the occurrence of different types of non-zero proximity out of any types of non-zero proximity among study participants. (B) The probability of the occurrence of different types of non-zero proximity during a day among study participants. 
largely due to the decreased of user pool and changed of class/dormitory arrangements. Next, we break down the probability by hours of the day as shown in Figure 5. Multiple combined proximity ( $\geq 2$ devices) occurs more during the day than in the night, especially around the noon hour. The single and none cases do not vary significantly across the day.

We next analyze whether the average active traffic is related to the combined proximity. The results are shown in Figure 6. The first four semesters show a similar pattern with the ones when only considering Bluetooth proximity. This is because Bluetooth proximity dominates the combined proximity since the WiFi proximity constitutes less than $10 \%$ of the combined proximity as shown in Figure 4A. However, as earlier mentioned, WiFi proximity dominates the combined proximity in semesters 5-6. As shown in Figure 6E and 6F, when multiple SMS contact/Facebook friend devices in proximity, the consumed traffic tends to decrease as well. Our results indicate that meaningful proximity is related to smartphone traffic usage regardless of which types of proximity we use. It is important to discern the social meaning of the proximity. In addition, WiFi proximity can be used as an extra measurement of proximity besides Bluetooth proximity.

\section{Big-Five personality traits}

For each group, we measure the average active traffic at different semesters as shown in Figure 7. Individuals that are more open/less neurotic/less conscientious tend to have more active traffic on average than individuals that are less open/more neurotic/more conscientious. More interestingly, individuals that were less agreeable/more extraverted
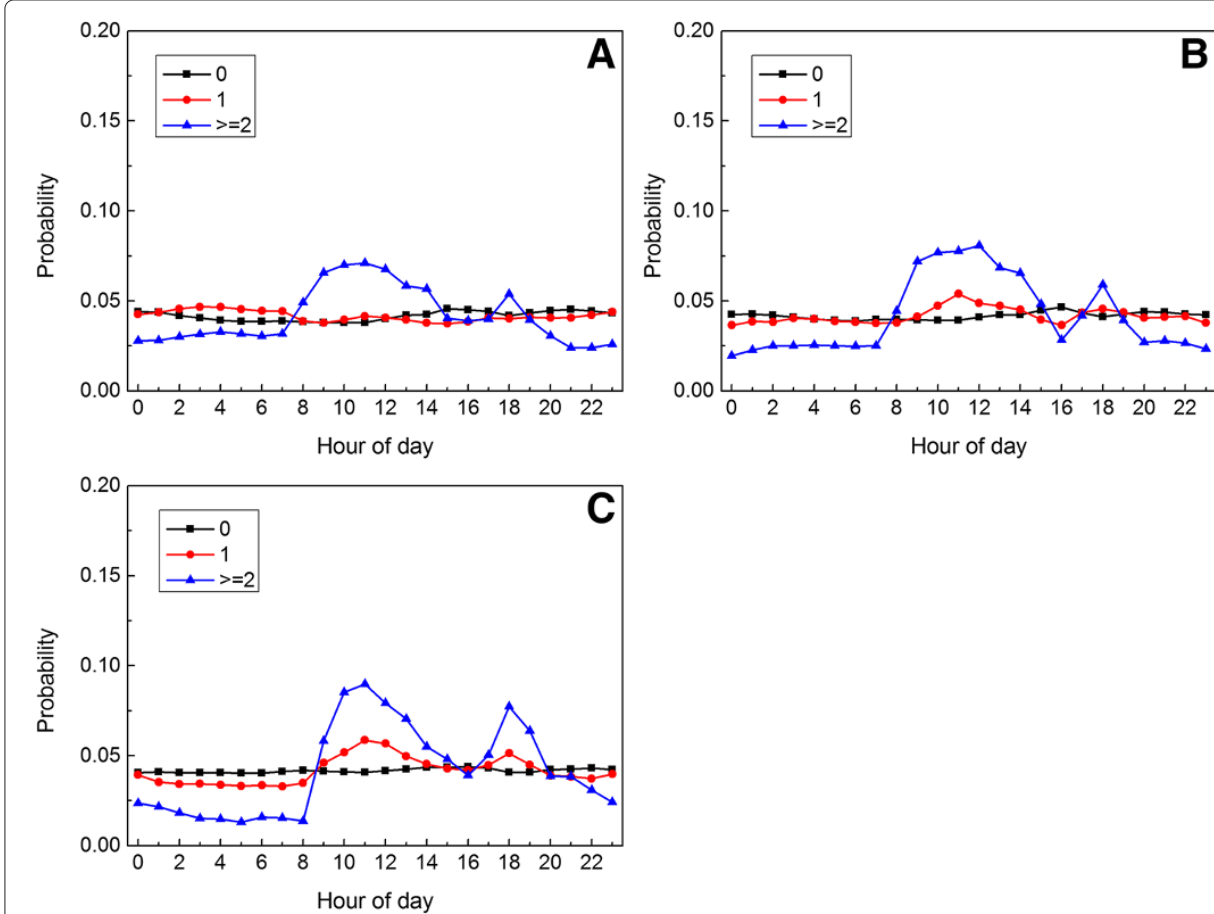

Figure 5 Diurnal patterns for different combined proximity densities. The diurnal patterns for the none (i.e., 0), single (i.e., 1), and multiple cases (i.e., $\geq 2$ ) at (A) semesters 1-2, (B) semesters 3-4, and (C) semesters 5-6. Each point represents the contribution of a given time slot with respect to that proximity category. 

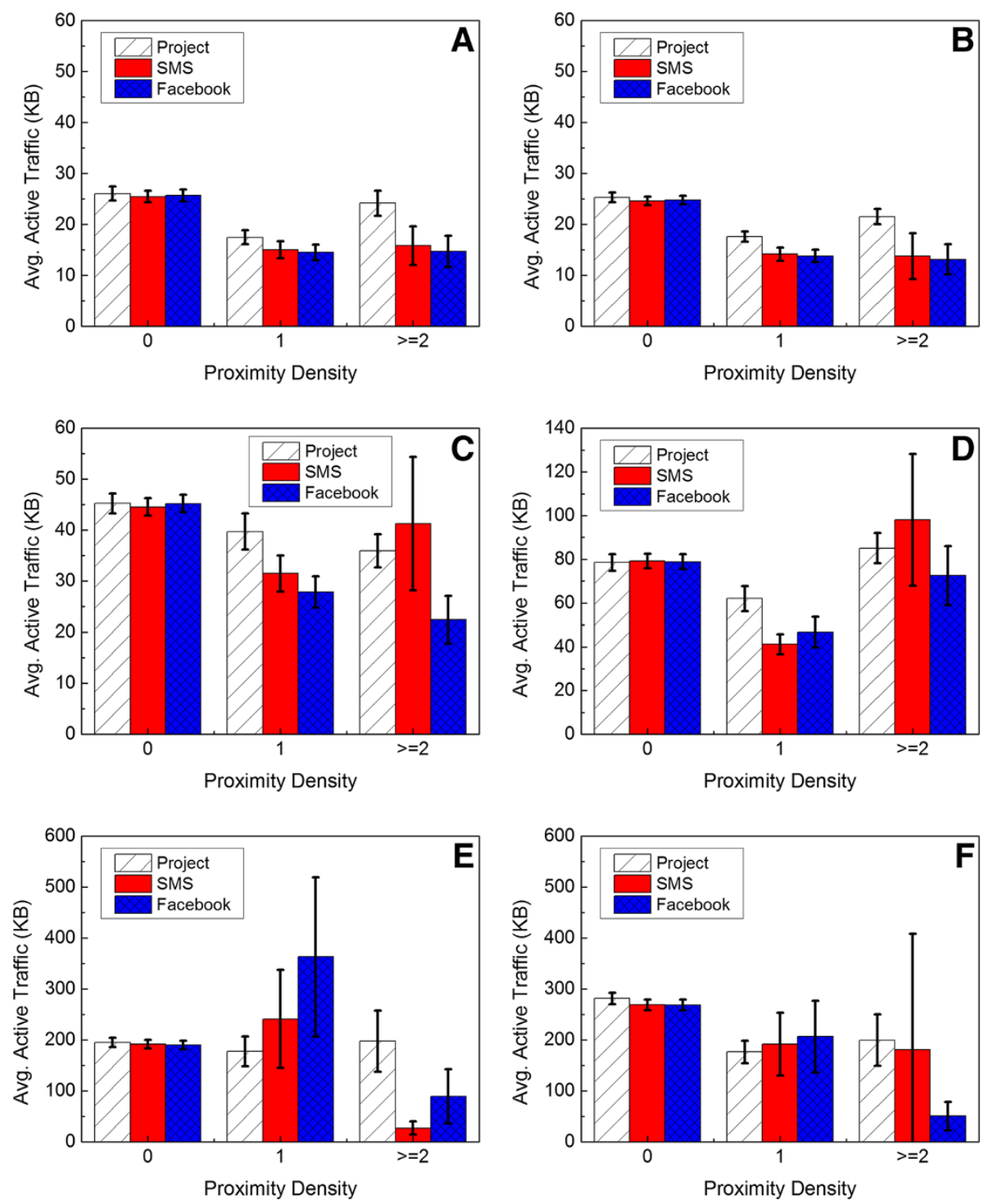

Figure 6 Average active traffic for different combined proximity densities. Four types of proximity are compared including any (i.e., any detected Bluetooth device), project (i.e., only NetSense devices via Bluetooth), SMS friends (i.e., send or receive at least one text message per month on average), Facebook friends (i.e., on Facebook friend list) at (A) semester 1, (B) semester 2, (C) semester 3, (D) semester 4, (E) semester 5, and (F) semester 6 .

tended to have more active traffic on average for the first four semesters but had less active traffic on average in the last two semesters.

In order to further understand whether individuals within the same group for extraversion and agreeableness change their smartphone usage behavior, we break down traffic by applications. Using extraversion as an example, we rank applications used in each group by their average active traffic and list the top five apps in Tables 2 and 3 for semesters 3 and 5 , respectively. We combine the groups with extraversion score equal to three and the groups with extraversion score less than three, since these two groups present a similar traffic usage pattern (Figure 7E). For each of the top ranked app, the average active traffic per day per person and the percentage of each app traffic out of total traffic consumed by 

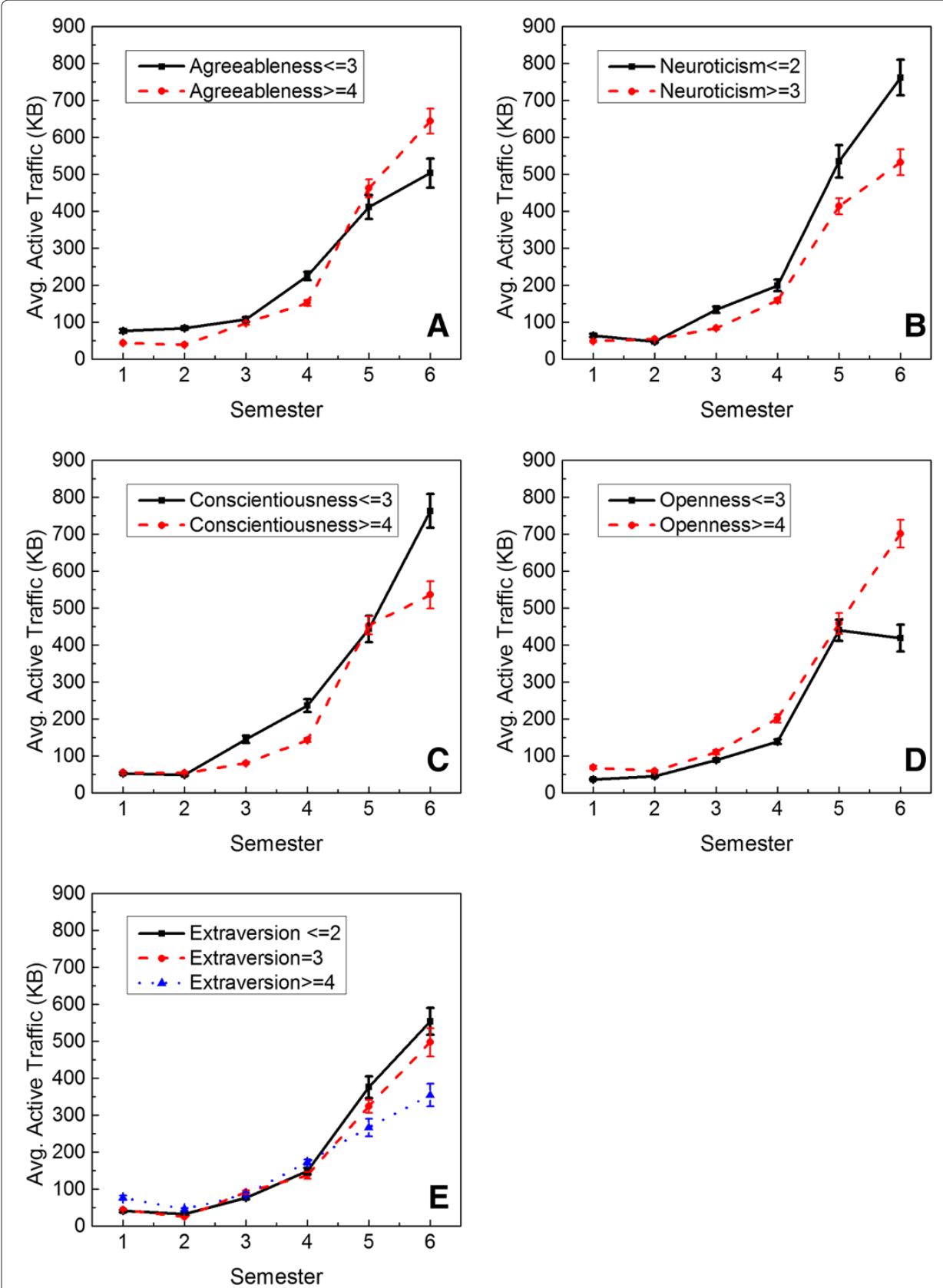

Figure 7 Average active traffic for Big-five personality traits. (A) Agreeableness. (B) Neuroticism. (C) Conscientiousness. (D) Openness. (E) Extraversion.

the group are also shown in the third and fourth columns, respectively. In semester 2, both more extraverted and less extraverted individuals shared similar apps such as Facebook, Android Media, Pandora, and Android Browser. However, more extraverted individuals consumed more traffic in these apps than less extraverted individuals. Less extraverted individuals tended to play more games such as Zynga Words while more extraverted individuals consumed significantly more Netflix. In other words, more extraverted individuals used more multimedia, social, and browser apps while less extraverted individual played more online games in semester 2 . In semester 6 , Facebook was used more by less 
Table 2 The top five apps ranked by the average active traffic (semester 3 )

\begin{tabular}{llll}
\hline & Apps & Average active traffic (KB) & Percentage (\%) \\
\hline (a) Extraversion $<=3$ & & & \\
1 & com.android.browser & 210.34 & 37 \\
2 & com.facebook.katana & 93.15 & 17 \\
3 & android.process.media & 43.82 & 8 \\
4 & com.pandora.android & 36.87 & 7 \\
5 & com.zynga.words & 16.95 & 3 \\
(b) Extraversion>=4 & & & \\
1 & com.netflix.mediaclient & $2,979.78$ & 75 \\
2 & com.android.browser & 257.86 & 7 \\
3 & com.pandora.android & 145.41 & 4 \\
4 & com.facebook.katana & 132.24 & 3 \\
5 & android.process.media & 76.99 & 2 \\
\hline
\end{tabular}

The average traffic is averaged over person and day.

extraverted individuals while Chrome, Stock Browser, and other browsers were used more by more extraverted individuals. In addition to Facebook, the less extraverted individuals also consumed more Youtube, Instagram and Snapchat. The results indicate that less extraverted people tend to use more multimedia and social apps than more extraverted people while the more extraverted people tended to use more browser apps.

It is also interesting that less extraverted individuals used more multimedia and social apps in semester 6 but less in semester 2. The main difference between the two semesters is that the participants used different types of handsets. The newer handsets in semester 6 have much faster network connectivity while the old handsets may restrict the usage of the phones [22].

\section{Location categories}

We analyze several representatives/popular applications including Facebook, the Android Browser, Entertainment and Sports Programming Network (ESPN), and Pandora as shown in Figures 8 and 9 for semesters 3 and 5, respectively. In semester 3, Android Browser tended to be used more in the classroom, especially if there was at least one device in proximity. For ESPN and Pandora, people tended to use more traffic in the dorm

Table 3 The top five apps ranked by the average active traffic (semester 5)

\begin{tabular}{llll}
\hline & Apps & Average active traffic (KB) & Percentage (\%) \\
\hline (a) Extraversion $<=3$ & & \\
1 & com.google.android.youtube & $4,333.92$ & 18 \\
2 & com.facebook.katana & $2,156.50$ & 9 \\
3 & com.android.chrome & $1,310.29$ & 6 \\
4 & com.instagram.android & 944.54 & 4 \\
5 & com.snapchat.android & 880.49 & 4 \\
(b) Extraversion $>=4$ & & 12 \\
1 & com.android.chrome & $2,402.86$ & 8 \\
2 & com.android.vending & $1,675.77$ & 8 \\
3 & com.facebook.katana & $1,595.45$ & 7 \\
4 & com.android.chrome(sandboxed) & $1,412.01$ & 7 \\
5 & com.sec.android.app.sbrowser & $1,353.59$ &
\end{tabular}




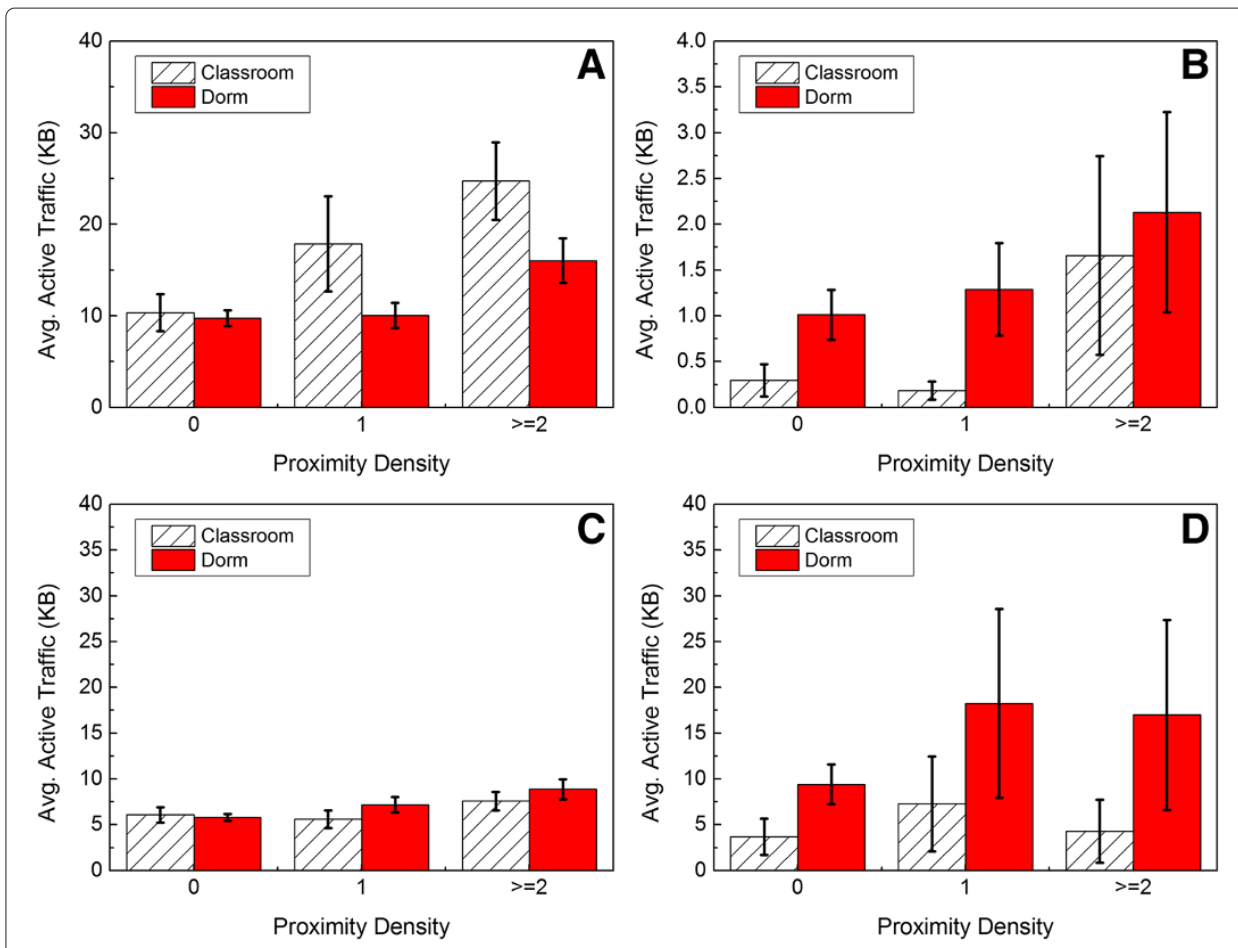

Figure 8 Location and combined proximity impact on app traffic at semester 3. (A) Browser. (B) ESPN. (C) Facebook. (D) Pandora.
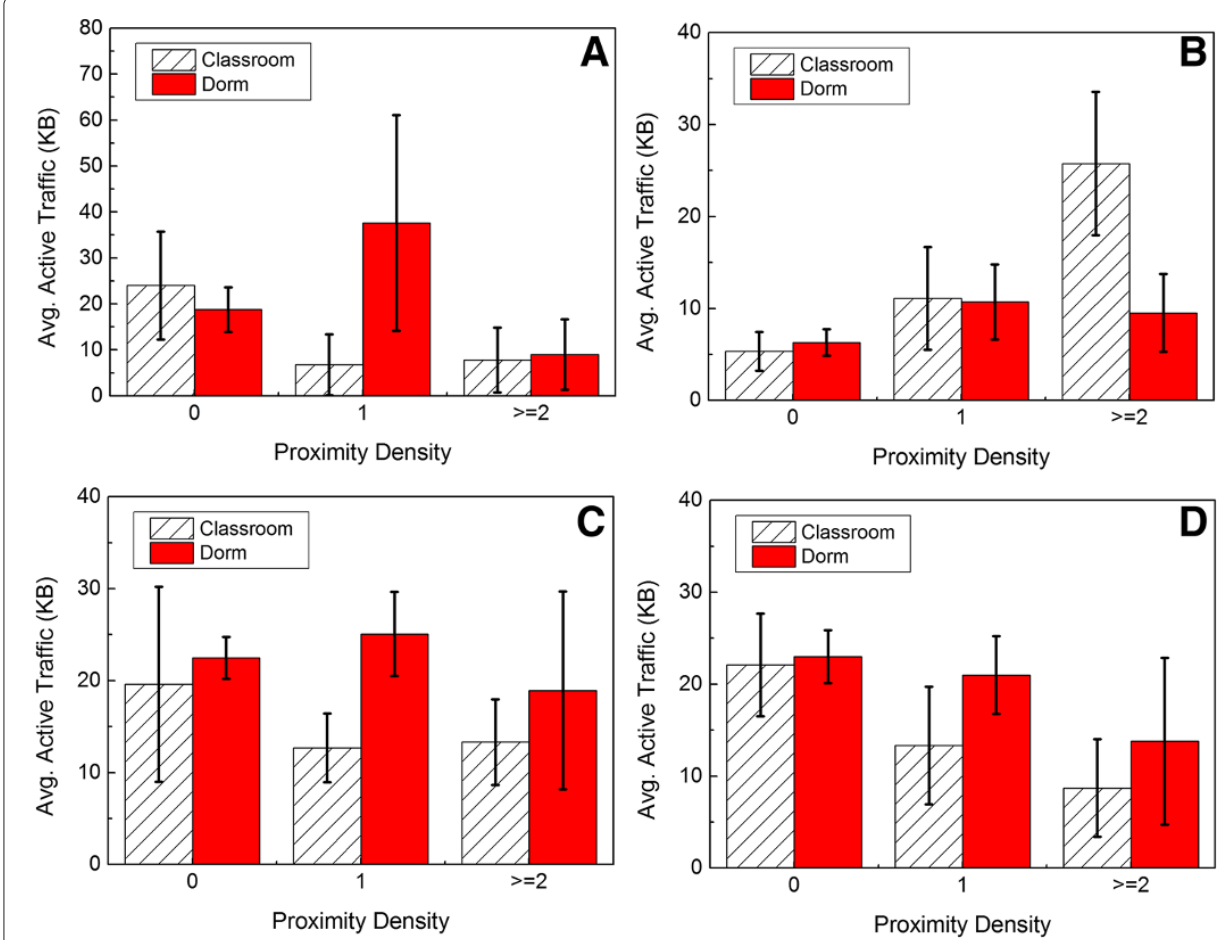

Figure 9 Location and combined proximity impact on app traffic at semester 5. (A) Browser. (B) ESPN (C) Facebook. (D) Pandora. 
especially when there were few people around. In semester 5, the Android Browser was used more in the dorm when there was a single device detected. For ESPN, it was used more in the classroom when multiple devices were around. For Facebook, there was no significant difference across location or proximity density at either semester since it is so popular among young people who use them everywhere they go. The same apps were not used similar at different semesters due to many possible reasons such as the popularity of the apps might have changed. For example, as mentioned earlier, the Android Browser was not used as often as earlier semesters but Chrome is used more instead. Another reason might be that the old handsets restricted the usage as discussed earlier.

\section{Conclusions}

In this paper, we analyze the longitudinal impact of proximity density, personality, and location on smartphone traffic consumption. Our results show that 1) friendship (i.e., SMS contacts and Facebook friendship) proximity has a significant impact on traffic consumption and 2) personality tends to impact application preference/consumption. However, the true behavior might not be properly captured due to the restrictions on network connectivity. We find that more extraverted individuals tends to use more multimedia and social apps than less extraverted individuals without the restriction of network connectivity, but the opposite when restricted; and 3) applications can have significantly different contextual usages based on the location, popularity of the app at the time, and network connectivity. We believe that our study raises the importance of the entirety of proximity, personality, and location context as future data relevant for the purposes of assessing user data consumption.

Future areas for improvement include explorations on larger population sizes, varying ages of participants, and non-campus locations.

Competing interests

The authors declare that they have no competing interests.

\section{Authors' contributions}

LM performed the computational data analysis and drafted the manuscript. SL helped developed the agent for data collection. AS supervised LM and SL in data analysis and paper writing. All authors read and approved the final manuscript.

\section{Acknowledgements}

We would like to thank our collaborators, Dr. Christian Poellabauer, Dr. David Hachen, and Dr. Omar Lizardo. Also, special thanks to Sprint who provided us more than two hundred phones and supported for the data plans making the data gathering a reality. This work was supported in part by the National Science Foundation through grant IIS-0968529 and Google.

Received: 1 July 2014 Accepted: 10 September 2014

Published online: 03 December 2014

References

1. Jin, Y, Duffield, N, Gerber, A, Haffner, P, Hsu, W-L, Jacobson, G, Sen, S, Venkataraman, S, Zhang, Z-L: Characterizing data usage patterns in a large cellular network. In: Proceedings of the 2012 ACM SIGCOMM Workshop on Cellular Networks: Operations, Challenges, and Future Design, August 13, 2012, Helsinki, Finland, pp. 7-12. ACM (2012)

2. Cisco: Cisco visual Cisco VNI Forecast, "Cisco Visual Networking Index: Global Mobile data Traffic Forecast Update 2009-2014". Cisco Public Information, February 9, 2010

3. Do, TMT, Blom, J, Gatica-Perez, J: Smartphone usage in the wild: a large-scale analysis of applications and context. In: Proceedings of the 13th International Conference on Multimodal Interfaces, Nov 14-18, 2012, Alicante, Spain, pp. 353-360. ACM (2011)

4. Karlsson, BF, Okada, K, Noleto, T: A mobile-based system for context-aware music recommendations. In: Artificial Intelligence Applications and Innovations, Vol. 382, Sept. 27-30, 2012, Halkidiki, Greece, pp. 520-529. Springer Berlin Heidelberg, (2012)

5. Chittaranjan, G, Blom, J, Gatica-Perez, D: Who's who with Big-Five: analyzing and classifying personality traits with smartphones. In: Wearable Computers (ISWC) 2011 15th Annual International Symposium on, June 12-15, 2011, San Francisco, CA, pp. 29-36. IEEE (2011) 
6. Blom, J, Chipchase, J, Lehikoinen, J: Contextual and cultural challenges for user mobility research. Commun. ACM 48(7), 37-41 (2005)

7. Eagle, N, Pentland, A: Reality mining: sensing complex social systems. Pers. Ubiquitous Comput. 10(4), 255-268 (2006)

8. Misra, A, Balan, RK: Livelabs: initial reflections on building a large-scale mobile behavioral experimentation testbed. ACM SIGMOBILE Mobile Comput. Commun. Rev. 17(4), 47-59 (2013)

9. Nandugudi, A, Maiti, A, Ki, T, Bulut, F, Demirbas, M, Kosar, T, Qiao, C, Ko, SY, Challen, G: Phonelab: a large programmable smartphone testbed. In: Proceedings of First International Workshop on Sensing and Big Data Mining, Nov 14, 2013, Roma, Italy, pp. 1-6. ACM (2013)

10. Zhang, Y, Årvidsson, A: Understanding the characteristics of cellular data traffic. ACM SIGCOMM Comput. Commun. Rev. 42(4), 461-466 (2012)

11. Falaki, H, Mahajan, R, Kandula, S, Lymberopoulos, D, Govindan, R, Estrin, D: Diversity in smartphone usage. In: Proceedings of the 8th International Conference on Mobile Systems, Applications, and Services, June 15-18, 2010, San Francisco, CA, pp. 179-194. ACM (2010)

12. Xu, Q, Erman, J, Gerber, A, Mao, Z, Pang, J, Venkataraman, S: Identifying diverse usage behaviors of smartphone apps. In: Proceedings of the 2011 ACM SIGCOMM conference on Internet measurement conference, Nov. 2-4, 2011, Berlin, Germany, pp. 329-344. ACM (2011)

13. Shafiq, MZ, Ji, L, Liu, AX, Pang, J, Wang, J: Characterizing geospatial dynamics of application usage in a $3 \mathrm{G}$ cellular data network. In: INFOCOM, 2012 Proceedings IEEE, March 25-30, 2012, Orlando, FL, pp. 1341-1349. IEEE (2012)

14. Rahmati, A, Zhong, L: Studying smartphone usage: lessons from a four-month field study. IEEE Trans. Mobile Comput. 12(7), 1417-1427 (2013)

15. Shin, $\mathrm{C}, \mathrm{Hong}, \mathrm{J}-\mathrm{H}, \mathrm{Dey}, \mathrm{AK}$ : Understanding and prediction of mobile application usage for smart phones. In: Proceedings of the 2012 ACM Conference on Ubiquitous Computing, Sept. 5-8, Pittsburgh, USA, pp. 173-182. ACM (2012)

16. Shi, K, Ali, K: GetJar mobile application recommendations with very sparse datasets. In: Proceedings of the 18th ACM SIGKDD international conference on Knowledge discovery and data mining, Aug. 12-16, Beijing China, pp. 204-212. ACM (2012)

17. Yan, T, Chu, D, Ganesan, D, Kansal, A, Liu, J: Fast app launching for mobile devices using predictive user context. In: Proceedings of the 10th International Conference On Mobile Systems, Applications, and Services, June 25-29, 2012, Low Wood Bay, Lake District, UK, pp. 113-126. ACM (2012)

18. Xu, Y, Lin, M, Lu, H, Cardone, G, Lane, N, Chen, Z, Campbell, A, Choudhury, T: Preference, context and communities: a multi-faceted approach to predicting smartphone app usage patterns. In: Proceedings of the 17th Annual International Symposium on International Symposium on Wearable Computers, Sept. 9-12, Zurich, Switzerland, pp. 69-76. ACM (2013)

19. Liu, S, Jiang, Y, Striegel, A: face-to-face proximity estimationusing bluetooth on smartphones. IEEE Trans. Mobile Comput. 13(4), 811-823 (2014)

20. Stopczynski, A, Sekara, V, Sapiezynski, P, Cuttone, A, Madsen, MM, Larsen, JE, Lehmann, S: Measuring large-scale social networks with high resolution. PloS one. 9(4), e95978 (2014)

21. Butt, S, Phillips, JG: Personality and self reported mobile phone use. Comput. Human Behav. 24(2), 346-360 (2008)

22. Striegel, A, Liu, S, Hu, X, Meng, L: LTE and WiFi: experiences with quality and consumption. In: Proceedings of the 11th International Conference on Mobile Systems and Pervasive Computing, Niagara Falls, Ontario, vol. 34, pp. 418-425, (2014)

23. Aharony, N, Pan, W, Ip, C, Khayal, I, Pentland, A: Social fMRI: investigating and shaping social mechanisms in the real world. Pervasive Mobile Comput. 7(6), 643-659 (2011)

24. Stopczynski, A, Larsen, JE, Lehmann, S, Dynowski, L, Fuentes, M: Participatory Bluetooth sensing: a method for acquiring spatio-temporal data about participant mobility and interactions at large scale events. In: Pervasive Computing and Communications Workshops (PERCOM Workshops) 2013 IEEE International Conference on, March 18-22, San Diego, USA, pp. 242-247. IEEE (2013)

25. Meng, L, Milenkovic, T, Striegel, A: Systematic dynamic and heterogeneous analysis of rich social network data. In: Complex Networks V, Vol. 549, pp. 25-37, (2014)

26. Catanese, S, De Meo, S, Ferrara, E, Fiumara, G, Provetti, A: Extraction and analysis of facebook friendship relations. In: Computational Social Networks, pp. 291-324. Springer, London (2012)

27. Dong, Y, Yang, Y, Tang, J, Chawla, NV: Inferring user demographics and social strategies in mobile social networks. In: KDD'14, New York, New York, pp. 15-24, (2014)

28. Carlotto, A, Parodi, M, Bonamico, C, Lavagetto, F, Valla, M: Proximity classification for mobile devices using wi-fi environment similarity. In: Proceedings of the First ACM International Workshop on Mobile Entity Localization and Tracking in GPS-less Environments, Sep 19, 2008, San Francisco, CA, pp. 43-48. ACM (2008) 